\title{
COMMUNITY STUDY OF MENSTRUAL IRON LOSS AND ITS ASSOCIATION WITH IRON DEFICIENCY ANAEMIA
}

\author{
BY \\ P. C. ELWOOD, M.D., D.P.H., D.C.H., AND G. REES, S.R.N., S.C.M. \\ From the Epidemiological Research Unit of the Medical Research Council \\ AND \\ J. D. R. THOMAS, M.Sc., F.R.I.C. \\ Senior Lecturer in Chemistry, Institute of Technology, University of Wales
}

Although anaemia, as conventionally defined, appears to be common in Great Britain (Kilpatrick, 1961; Elwood, 1964), little is known about the factors which are likely to cause a negative iron balance and eventually lead to iron deficiency anaemia. The main loss of iron from the body in females is by menstruation, but the relative importance of menstrual loss and dietary intake of iron in the determination of the level of circulating haemoglobin does not seem to have been examined in a representative sample of the community.

A series of epidemiological studies was conducted recently to investigate a variety of aspects of iron deficiency. The basic study is described in Elwood, Waters, Greene, and Wood (1967). In the study reported here, the association between haematological state and menstrual loss of iron is examined in a representative sample drawn from a total community. Details of the dietary intake of iron in these women will be presented elsewhere but the relevance to iron balance of both menstrual loss and dietary intake is examined.

\section{Method}

An area in the Rhondda Fawr was selected and the 1,005 female residents aged 20 to 64 years were invited to co-operate in a haematological survey. Women who were pregnant were omitted. The 355 women who were between 20 and 39 years inclusive were stratified by haemoglobin level at intervals of $1 \mathrm{~g} . / 100 \mathrm{ml}$. blood, and within these groups sub-samples of up to eleven subjects were chosen at random to give a total sample of 59 (Table I). Of these, four who had become pregnant since the initial survey were excluded, as were a further six whose menstrual periods occurred at such irregular intervals that estimates of the iron lost in one or two menstrual periods could not be used as a guide to the loss over long periods of time.

Each woman was visited by a doctor and asked to co-operate in the present study. Those who agreed were visited by a health visitor (G.R.) and the method for collection of the menstrual loss was explained. "Soluble" pads or tampons of a single make were supplied throughout the study.

TABLE I

DISTRIBUTIONS BY HAEMOGLOBIN LEVEL OF THE SAMPLE AND THE POPULATION FROM WHICH THEY WERE DRAWN

\begin{tabular}{|c|c|c|c|c|c|c|}
\hline \multirow{2}{*}{$\begin{array}{l}\text { Hb Level } \\
\text { (g./100 ml.) }\end{array}$} & \multirow{2}{*}{$\begin{array}{c}\text { Total } \\
\text { Population } \\
\text { (20-39 yrs) }\end{array}$} & \multirow{2}{*}{ Sample Drawn } & \multicolumn{2}{|c|}{ Excluded } & \multirow{2}{*}{ Refused } & \multirow{2}{*}{ Examined } \\
\hline & & & Pregnant & Unsuitable & & \\
\hline $8-$ & 3 & 3 & - & 1 & - & 2 \\
\hline 9- & 1 & 1 & - & - & - & 1 \\
\hline 10 & 12 & 11 & - & 1 & - & 10 \\
\hline $11-$ & 18 & 11 & - & 1 & 3 & 7 \\
\hline $12-$ & 46 & 11 & 1 & 2 & - & 8 \\
\hline 13- & 161 & 11 & 2 & 1 & 1 & 7 \\
\hline $14+$ & 114 & 11 & 1 & - & 1 & 9 \\
\hline Total & 355 & 59 & 4 & 6 & 5 & 44 \\
\hline
\end{tabular}


Immediately after use the woman placed these in a 5 litre plastic container with 3 litres of de-ionized water. The women who worked away from home were given plastic bags and asked to keep in them all pads or tampons removed when away from home until they could be put in the container. A wooden stick was placed in the container to enable the pads to be immersed in the water. The need to obtain as complete a collection as possible was repeatedly stressed and after each collection each woman was questioned about how much blood appeared to have escaped.

On completion of the menstrual period the container was collected and mechanically agitated until the pads and all clots had disintegrated. The contents of the container were carefully inspected for evidence of clots, and if satisfactory in this respect, an aliquot of the fluid was removed. A $25-\mathrm{ml}$. aliquot (or, where the blood loss had been heavy, a 10-ml. aliquot) was evaporated to dryness over a low Bunsen burner and then ashed at a temperature below $550^{\circ} \mathrm{C}$. for under $10 \mathrm{~min}$. The ashed sample was treated with $1 \mathrm{ml}$. concentrated hydrochloric acid $+1 \mathrm{ml}$. concentrated nitric acid, evaporated to dryness, further treated with $2 \mathrm{ml}$. concentrated hydrochloric acid, and again evaporated to dryness. The sample was then transferred to a graduated flask, using de-ionized water and 4 or 5 drops of concentrated hydrochloric acid, and the volume of the extract was made up to $10 \mathrm{ml} ; 2 \mathrm{ml}$. of this extract were treated with $2 \mathrm{ml}$. de-ionized water, $0.5 . \mathrm{ml} .20$ per cent. citric acid solution, 2 drops of thioglycollic acid, and $0.5 \mathrm{ml} .0 .880$ ammonium hydroxide. The optical density of the resulting solution was measured at $520 \mu$. and compared with a calibration curve obtained for solutions containing standard amounts of iron. The iron loss is expressed here in terms of milligrams of iron lost during the complete period. No estimates of the volume of blood lost were made as this seems to be a confusing way of presenting the data. As Barer and Fowler (1938) pointed out, any loss of blood between say 33 and $100 \mathrm{ml}$. may represent $5 \mathrm{~g}$. haemoglobin depending on the level of haemoglobin in the circulating blood.

In order to obtain more precise estimates of the average iron loss for each woman, they were all asked to co-operate a second time but eight declined to do so. Three who showed in the two periods large differences were asked to co-operate on a third occasion. In addition, a random sample of twelve women was asked to co-operate on six occasions, but only six agreed to do so, though two co-operated on five occasions. In the analyses which follow, the mean of all the available deter- minations for each woman is used as the estimate of her loss, but in four cases data relating to a menstrual loss which was said to have been incompletely collected were omitted. One of these omissions was the collection for one period of a woman who commenced taking oral contraceptives during the study, and whose loss fell to about half what it had been. All the other women denied taking oral contraceptives.

In addition to the estimate of mean menstrual loss, an estimate of the haemoglobin level (estimated as cyanmethaemoglobin and stated as $\mathrm{g}$. $\mathrm{Hb} / 100 \mathrm{ml}$. blood) is available for each woman, based on a venous sample of blood taken during the preliminary haematological survey conducted a few months before the present study.

It has been shown that menstrual loss of blood is decreased when a woman is iron deficient and correction of the deficiency leads to an increase in menstrual loss (Jacobs and Butler, 1965). On the other hand Taymor, Sturgis, and Yahia (1964) argue that anaemia can cause menorrhagia. In the examination of the association between iron deficiency and menstrual blood loss it therefore seems more reasonable to use as an estimate of a woman's iron loss from menstruation a measurement made when she is not iron deficient, that is, at a time when any compensatory mechanism in iron defis ciency is not in operation. Therefore the measurements of menstrual iron loss were all made after correction of the haemoglobin level of the women who had low levels when first seen. The haemoglobin levels of all of these were brought up to at least $11.5 \mathrm{~g}$. and almost all above $12.0 \mathrm{~g}$. before their first measurement of menstrual loss. However the estimate of the haematological state of each woman was based on the haemoglobin level found at the initial survey. This latter is considered to give a reasonable estimate of the degree of iron imbalance in the individual women, as the circulating haemoglobin level of most had probably reached a reasonably steady state during the years before examination in our initial screening survey.

\section{Results}

The distributions by haemoglobin level of the total population and of the sample for the present study are shown in Table I. Because of exclusions the effective sample size was 49 women, and of these five refused to co-operate. Data are therefore available for 44 women ( 90 per cent: of the 49 eligible), and the results which follow are based on 110 measurements in these women.

The consistency of the loss within women was assessed by the coefficient of variation. In this 
calculation the standard error was based on the residual variance after the component attributable to differences between women had been eliminated. The coefficient of variation is 54 per cent. Reexamination of data presented by Hytten, Cheyne, and Klopper (1964) relating to fifteen healthy midwives and 23 "normal" parous women gives a coefficient of 29 per cent. Similar data presented by Hallberg and Nilsson (1964) relating to twelve healthy probationer nurses aged 21-23 years gives a coefficient of 31 per cent., and data presented by Baldwin, Whalley, and Pritchard (1961), who studied 21 volunteers, chiefly nurses, all of whom considered themselves to have normal menstrual loss, show a coefficient of 35 per cent.

The mean loss per menstrual period in the group is $12.17 \pm 1.67 \mathrm{mg}$. iron and this is similar to previous published series. However, reference to the mean of a distribution as skewed as this is likely to be misleading, and the omission of extreme values, as has been done by some authors, is a very dubious procedure. We have preferred to reduce skewness by transformation of data and have used logarithms (to base 10). This transformation greatly reduces the deviations from a Gaussian distribution, and they are no longer statistically significant $(0 \cdot 1>P>0 \cdot 05)$. In transformed units the mean loss is $1.0111 \pm 0.06$; the mean loss in original units was $10.3 \mathrm{mg}$. iron, with the 95 per cent. confidence limits of the mean as 12.6 and $8.0 \mathrm{mg}$.

Table II shows the distribution of the subjects by mean menstrual loss, parity, and age. There is no obvious association between the distributions. Again the data were transformed and the mean of each subgroup compared. This confirmed that there is no evidence of a significant (at $\mathrm{P}<0.05$ ) effect of age or parity on menstrual iron loss.

The association between the level of circulating haemoglobin at the time of the screening survey and menstrual iron loss is examined in Table III.

TABLE III

\begin{tabular}{|c|c|c|c|c|c|c|c|c|}
\hline \multirow{2}{*}{$\begin{array}{l}\text { Total Iron } \\
\text { Loss (mg.) }\end{array}$} & \multicolumn{8}{|c|}{ Haemoglobin Level (g./100 ml.) } \\
\hline & 8- & 9- & 10 & $11-$ & 12- & 13 & $14+$ & Total \\
\hline$<4$ & - & - & 1 & 2 & 2 & 3 & 3 & 11 \\
\hline 4 & - & - & - & 2 & 4 & 2 & 1 & 9 \\
\hline 8- & 一 & 一 & - & 一 & - & - & 1 & 1 \\
\hline $12-$ & - & 1 & 2 & 一 & - & 1 & 1 & 5 \\
\hline $16-$ & 2 & 一 & - & - & 2 & - & 1 & 5 \\
\hline 20 & - & 一 & 3 & 1 & - & 1 & - & 5 \\
\hline 24 & - & - & 1 & - & - & - & - & 1 \\
\hline 28- & - & - & 2 & 2 & - & - & 1 & 5 \\
\hline $32-$ & - & - & - & - & - & - & - & - \\
\hline $36+$ & - & - & 1 & - & - & - & 1 & 2 \\
\hline Total & 2 & 1 & 10 & 7 & 8 & 7 & 9 & 44 \\
\hline
\end{tabular}

There is some evidence of a negative associationin general the women with lower haemoglobin levels have higher menstrual losses. Following transformation, as above, the strength of this association was assessed by derivation of a correlation coefficient $(r)$. This shows that the association is significant $(r=-0.37 ; t=2.73 ; 0.01>\mathrm{P}>$ 0.001 ) and suggests that about 14 per cent. of the

TABLE II

DISTRIBUTION OF WOMEN BY MENSTRUAL IRON LOSS, PARITY, AND AGE GROUP

\begin{tabular}{|c|c|c|c|c|c|c|c|c|c|c|c|c|c|}
\hline \multirow{2}{*}{\multicolumn{2}{|c|}{$\begin{array}{l}\text { Parity } \\
\text { Age (yrs) }\end{array}$}} & \multicolumn{2}{|c|}{0} & \multicolumn{2}{|c|}{$1+2$} & \multicolumn{2}{|c|}{$3+$} & 0 & $1+2$ & $3+$ & \multicolumn{2}{|c|}{ All Parities } & \multirow[b]{2}{*}{ Total } \\
\hline & & $20-$ & $30-$ & 20 & $30-$ & 20 & $30-$ & & $1 \mathrm{Ag}$ & & 20 & 30 & \\
\hline \multirow{10}{*}{$\begin{array}{l}\text { Total } \\
\text { Iron } \\
\text { Loss } \\
\text { (mg.) }\end{array}$} & $<4$ & - & 2 & - & 2 & 2 & 2 & 2 & 2 & 4 & 2 & 6 & 8 \\
\hline & 4 & 1 & - & 2 & 4 & 2 & 2 & 1 & 6 & 4 & 5 & 6 & 11 \\
\hline & $8-$ & - & - & 1 & 1 & - & - & - & 2 & - & 1 & 1 & 2 \\
\hline & $12-$ & 1 & 1 & - & 1 & 1 & 1 & 2 & 1 & 2 & 2 & 3 & 5 \\
\hline & $16-$ & - & - & 1 & 1 & - & 3 & - & 2 & 3 & 1 & 4 & 5 \\
\hline & $20-$ & 1 & - & - & 1 & - & 4 & 1 & 1 & 4 & 1 & 5 & 6 \\
\hline & 24 & - & - & - & 1 & - & - & - & 1 & - & - & 1 & 1 \\
\hline & $28-$ & - & - & - & 1 & 1 & 1 & - & 1 & 2 & 1 & 2 & 3 \\
\hline & $32-$ & - & 1 & 一 & - & - & - & 1 & - & - & - & 1 & 1 \\
\hline & $36-$ & - & 1 & - & - & - & 1 & 1 & - & 1 & - & 2 & 2 \\
\hline \multicolumn{2}{|l|}{ Total } & 3 & 5 & 4 & 12 & 6 & 14 & 8 & 16 & 20 & 13 & 31 & 44 \\
\hline
\end{tabular}


variation in haemoglobin level is dependent on menstrual iron loss.

It has been shown that in young women the level of circulating haemoglobin is significantly associated with body weight (Elwood, Kilpatrick, and Withey, 1964), and it can reasonably be assumed that it is ultimately dependent on the dietary intake of iron. An estimate of the intake of iron of each woman was made from a one week weighed record. The inter-relationships of the mean total daily intake of iron in mg. (diet $\mathrm{Fe}$ ), mean transformed iron loss in mg. in one menstrual period (log. menses $\mathrm{Fe}$ ), body weight in $\mathrm{kg}$. (wt), age in years, and circulating haemoglobin in $\mathrm{g} . / 100 \mathrm{ml}$. blood (Hb) can be summarized thus:

$\mathrm{Hb}=11.77-0.0155 \mathrm{age}+0.0519 \mathrm{wt}-0.464 \operatorname{diet} \mathrm{Fe}$ -1.5909 Log. menses Fe

In this equation only the contribution from menstrual loss is clinically important and statistically significant, though the contribution from body weight just reaches statistical significance.

\section{Discussion}

We believe this is the first study to be published in which data relating to menstrual iron loss in a sample representative of the community is reported. Several authors have presented results for "normal" women, usually hospital patients or nurses, and have attempted to estimate from them the "average daily loss" for women in the community. However, the definition of "normal subjects" in these studies has been arbitrary and hospital patients can never be assumed to represent the community. Furthermore, the use of volunteer nurses or midwives in a study such as this seems to be unwise. Considerable self-selection is likely to occur in nurses, and especially midwives, as those whose menstrual loss is unusually light, heavy, or irregular in amount may consider themselves unsuitable for inclusion in such studies. Estimates of means and dispersion, and of consistency within subjects and associations with other variates may therefore be biased if based on such subjects. Population parameters can only be assessed from samples representative of the community.

However, in addition to sources of error in the sample, errors can all too easily be introduced by the incomplete collection of menstrual loss by the individual subjects. The fact that the mean loss in the present study is slightly lower and the coefficient of variation higher than in most of the studies previously reported suggests that some of our subjects may not have collected their complete loss. The need for complete collection was repeatedly stressed, and each woman was questioned about $\triangle$ this after each period measured. While it is possible that our estimate of the mean loss is low, it seems unlikely that it is greatly in error. It is not unreasonable to assume that collection on the second $\stackrel{\vec{\rho}}{\stackrel{2}{ }}$ occasion by each woman was more complete than on the first occasion, as the questioning by the nurse following the first measurement would emphasize the need for complete collection. In fact, omitting the five women who reported incomplete collection on the first occasion and whose first results have been omitted from the results already presented, the mean of the first collection in the women who co-operated on two occasions is 13.5 mg., which is only just less than the mean of the subsequent collections in these women, $13.7 \mathrm{mg}$. A similar trend, also statistically insignificant, is $N$ shown in the data of Schlaphoff and Johnston $\omega$ (1949). Nevertheless it is possible that our estimate $\vec{N}$ of the mean loss is low. If it is, this is likely to be due to incomplete collection by women with the higher losses, and our data may then under- $\vec{c}$ estimate the relationship between haemoglobin level and menstrual loss.

Many authors have assumed that menstrual loss of iron is an important aetiological factor in irom deficiency. Jacobs and Butler (1965) compared the menstrual blood loss in a group of "norma? volunteer midwives with that of a group of hospital patients with iron deficiency anaemia, and found a

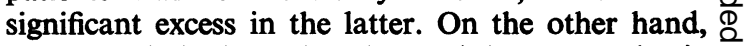
Haden and Singleton (1933) stated that menorrhagia $\underset{\vec{F}}{\overrightarrow{2}}$ is not responsible for iron deficiency, though $\frac{0}{3}$ they presented no data directly supporting this statement, and Barer and Fowler (1936) and Rankin, Veall, Huntsman, and Liddell (1962) stated that they found no convincing evidence of an $\stackrel{D}{\square}$ association between blood loss and haemoglobin level. Millis (1951) stated that his subject showed 3 . no consistent relationship between haemoglobin 8 levels and the duration of the menstrual cycle, and Barer and Fowler (1938) stated that the degree of 0 anaemia did not parallel the amount of blood lost. Barer and Fowler (1938) presented data for a $\frac{D}{2}$ rather ill-defined group of eighteen women who either complained of menorrhagia or had been $N$ admitted to hospital with hypochromic anaemia N which seemed to be partly attributable to menstrual N loss even when the women thought their losses $\frac{\omega}{\sigma}$ were normal. These data have been re-examined and found to suggest the unexpected conclusion $\stackrel{0}{\odot}$

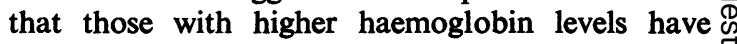
higher menstrual losses $(r=0 \cdot 43)$. Data presented by Millis (1951) for fourteen nurses suggest the same conclusion $(r=0.28)$ though in neither these data 
nor in those of Barer and Fowler (1938) is the trend statistically significant at $\mathrm{P}<0.05$.

There is therefore considerable conflict in the evidence presented and the conclusions drawn in previously published studies. There is further conflict in the hypotheses advanced by different authors on the nature of the association between menstrual loss and iron deficiency. Jacobs and Butler (1965) maintained that there operated in iron deficiency a compensatory mechanism which tended to decrease menstrual loss. They presented data to support this as they showed that a group of anaemic women had an increased menstrual blood loss after correction of their iron deficiency. On the other hand, Taymor and others (1964) argued that anaemia could be the cause of menorrhagia, though they appeared to have made no measurements of menstrual loss in their studies. If the arguments of these authors are true, though they do not seem reasonable in view of the data presented by Jacobs and Butler (1965), then we have underestimated the strength of the association between menstrual iron loss and haemoglobin level, as the iron deficiency of all our subjects had been corrected before their menstrual losses were measured. However, even if anaemia can cause menorrhagia in some women, and even if a vicious cycle can be set up, as these authors postulate, it still seems to us that the association between haemoglobin level and menstrual loss of greatest interest is that which exists in the absence of the added effect of any such vicious cycle, and, furthermore, in the absence of the effect of any compensatory mechanism such as that suggested by Jacobs and Butler (1965). The fact that we found a clinically important and statistically significant association between haemoglobin level and menstrual loss, after the correction of the iron deficiency in our subjects, seems to us to establish that menstrual loss plays a part in the development of iron deficiency rather than vice versa. Furthermore, the strength of the association we have detected suggests that it is a part of clinical importance. It is of interest that the relevance of total dietary intake of iron to iron balance appears to be much smaller, but we have discussed this further elsewhere (Elwood, 1967).

The striking lack of agreement in the data presented in the literature and in the conclusions and hypotheses based on them seems to us to arise largely from the use of unsatisfactory samples. Hospital patients and volunteer nurses can never be assumed to represent the community, and the factors involved in their selection can undoubtedly introduce serious bias. Furthermore, the use of groups of "normal" and "iron-deficient" subjects which are discrete with regard to their distributions by haemoglobin level is inefficient, for data from such groups cannot be used to estimate the strength of the relationship between variates such as haemoglobin level and menstrual loss. Haemoglobin level is best considered as a variable with a continuous distribution and the association between it and other variates, such as menstrual loss, is best examined by a regression or correlation technique.

\section{SUMmary}

A study is described of the association between menstrual iron loss and the level of circulating haemoglobin in a community sample of women. The association was found to be clinically important and statistically significant. The data are compared with those published by other authors.

\section{REFERENCES}

Baldwin, R. M., Whalley, P. J., and Pritchard, J. A. (1961). Amer. J. Obstet. Gynec., 81, 739 (Measurement of menstrual blood loss).

Barer, A. P., and Fowler, W. M. (1936). Ibid., 31, 979 (The blood loss during normal menstruation).

- (1938). Ibid., 35 839, (The blood loss in menorrhagia).

Elwood, P. C. (1964). Brit J. prev. soc. Med., 18, 81 (Distribution of haemoglobin level, packed cell volume and mean corpuscular haemoglobin concentration in women in the community).

(1967). Communication to Symposium on "Occurrence, Causes, and Prevention of Nutritional Anaemias." Swedish Nutrition Foundation (The role of food iron in the prevention of anaemia).

- Kilpatrick, G. S., and Withey, J. L. (1964). Brit. J. prev. soc. Med., 18, 125 (Distribution of haemoglobin level in a group of schoolgirls and relation to height, weight, and other variables).

, Waters, W. E., Greene, W. J., and Wood, M. M. (1967). Brit. med. J., 14, 714 (Evaluation of a screening survey for anaemia in adult non-pregnant women).

Haden, R. L., and Singleton, J. M. (1933). Amer. J. Obstet. Gynec., 26, 330 (Distribution of menstruation due to simple achlorhydric anaemia).

Hallberg, L., and Nilsson, L. (1964). Acta obstet. gynaec. scand., 43, 352 (Constancy of individual menstrual blood loss).

Hytten, F. E., Cheyne, G. A., and Klopper, A. I. (1964). J. Obstet. gynaec. Brit. Cwlth, 71, 255 (Iron loss at menstruation).

Jacobs, A., and Butler, E. B. (1965). Lancet, 2, 407 (Menstrual blood loss in iron deficiency anaemia).

Kilpatrick, G. S. (1961). Brit. med. J., 2, 1736 (Prevalence of anaemia in the general population).

Millis, J. (1951). Med.J. Aust., 2, 874 (The iron losses of healthy women during consecutive menstrual cycles).

Rankin, G. L. S., Veall, L. N., Huntsman, R. G., and Liddell, J. (1962). Lancet, 1, 567 (Measurement with $51 \mathrm{Cr}$. of red-cell loss in menorrhagia).

Schlaphoff, D., and Johnston, F. A. (1949). J. Nutr., 39, 67 (The iron requirements of six adolescent girls).

Taymor, M. L., Sturgis, S. H., and Yahia, C. (1964). J. Amer. med. Ass., 187, 323 (The etiological role of chronic iron deficiency in production of menorrhagia). 